\title{
Stepped Care Treatment of Postpartum Depression: Impact on Treatment, Health, and Work Outcomes
}

\author{
Dwenda Gjerdingen, MD, MS, Scott Crow, MD, Patricia McGovern, PhD, MPH, \\ Michael Miner, PhD, and Bruce Center, PhD
}

Purpose: The purpose of this study was to pilot a stepped collaborative care intervention for women with postpartum depression and evaluate health differences between self-diagnosed depressed and nondepressed women.

Methods: Five hundred six mothers of infants from 7 clinics completed surveys at 0 to 1, 2, 4, 6, and 9 months postpartum and a Structured Clinical Interview for DSM-IV (SCID). SCID-positive depressed women were randomized to stepped collaborative care or usual care. Nine-month treatment, health, and work outcomes were evaluated for stepped care women $(n=19)$ versus control depressed women $(n=20)$, and self-diagnosed depressed women $(n=122)$ versus nondepressed women $(n=344)$.

Results: Forty-five women had SCID-positive depression whereas 122 had self-diagnosed depression. For SCID-positive depressed women, the stepped care intervention increased mothers' awareness of their depression diagnosis $(100 \%$ vs $61 \% ; P=.008)$ and their receipt of treatment $(94 \%$ vs $56 \% ; P=$ .019). Self-diagnosed depressed women (vs nondepressed women) had more depressive symptoms and acute care visits, worse general and mental health, and greater impact of health problems on regular activities.

Conclusions: The stepped care intervention improved women's knowledge of their postpartum depression diagnosis and their receipt of treatment. However, our formal diagnostic procedures missed many women whose depressed mood interfered with their health and function. (J Am Board Fam Med 2009;22:473-482.)

Postpartum depression (PPD), the most prevalent serious complication of pregnancy, affects up to $22 \%$ of women who have recently given birth. ${ }^{1}$ Given that fewer than half of postpartum women are screened for depression, ${ }^{2-5}$ it follows that only a minority of cases are probably treated.

This article was externally peer reviewed.

Submitted 11 September 2008; revised 13 December 2008; accepted 26 December 2008.

From the Department of Family Medicine and Community Health (DG, MM, BC), the Department of Psychiatry (SC), and the School of Public Health (PM), University of Minnesota, St. Paul.

Funding: This study was funded by the National Institute of Mental Health.

Conflict of interest: none declared.

Corresponding author: Dwenda Gjerdingen, MD, MS, 580 Rice St, St. Paul, MN 55103 (E-mail: dgjerdin@ umphysicians.umn.edu).

\footnotetext{
See Related Commentary on Page 471.
}

In studies of nonpostpartum depression, collaborative treatment of depression has been acclaimed as superior to traditional treatment methods (eg, antidepressants and/or psychotherapy). Collaborative care consists of a team-based approach to care that, when applied to mental disorders, involves a primary care provider, mental health specialist (psychiatrist and/or psychologist or social worker), care manager, ${ }^{6-11}$ and other allied health professionals, as needed. The team usually offers enhanced provider/patient education about major depression, treatment with antidepressant medication and/or psychotherapy, and regular monitoring by the care manager (often by telephone) of depressed patients' symptoms and treatment adherence. ${ }^{7} \mathrm{~A}$ recent meta-analysis of 37 collaborative care studies documented that collaborative care, compared with usual depression care, improves depression treatment adherence and outcomes, with long-term benefits of at least 5 years. ${ }^{12}$

Collaborative care treatment programs can improve not only depressive symptoms ${ }^{6,7,10,13,14}$ 
but also depressed patients' function, ${ }^{7,14,15}$ quality of life, ${ }^{7}$ pain, ${ }^{14}$ work performance and productivity, ${ }^{16,17}$ marital adjustment, ${ }^{18}$ and physical health, including chronic health conditions such as arthritis. ${ }^{14}$

A special type of collaborative care-stepped care treatment-delivers care in a step-wise manner, beginning with screening, diagnosis, and initial treatment in a primary care setting and adding follow-up and support, decision support, mental health consultation, or referral by a care manager as needed for patients with persistent depressive symptoms. ${ }^{19}$ Although it would seem that collaborative care would also improve PPD outcomes, neither collaborative or stepped care have ever been experimentally tested in a postpartum population. Such evaluation would be important given that postpartum women often possess unique barriers to treatment, such as the need for childcare during mental health visits, ${ }^{20}$ concerns about medication effects on nursing infants, ${ }^{21}$ and fear of judgment or referral to child protection. ${ }^{22}$

The purpose of this study was to pilot a stepped collaborative care treatment program for women with PPD to begin to test the impact of such a program on women's mental and physical health outcomes and to evaluate differences in health outcomes between women with and without PPD.

\section{Methods}

\section{General Procedures}

Before its initiation the study was approved by the University of Minnesota's Institutional Review Board. Patients were recruited for the study from October 1, 2005, through September 30, 2006, during well-child visits, and surveys were administered from October, 2005, through June, 2007. Mothers registering their infants for an initial (0 to 1 month) well-child visit at one of 7 participating clinics were given an enrollment packet by the receptionist. The packet consisted of a brief description of the study, an enrollment form (indicating their willingness to participate), a consent form, and an initial survey. Enrollees were given follow-up surveys at subsequent 2-, 4-, and 6-month well-child visits (or, alternatively, they completed telephone or mailed surveys), and were mailed a final survey at 9 months postpartum. Mothers were also asked to complete the depression module of the Structured Clinical Interview for DSM-IV
$(\mathrm{SCID})^{23}$ within 2 weeks of the 0 - to 1 -month survey, and subsequently if a previously nondepressed woman developed either a positive 2-question depression screen or 9-item Patient Health Questionnaire (PHQ-9) at a later interval.

\section{Study Participants and Practices}

Participants were recruited from 7 Minneapolis/St. Paul metropolitan area clinics: 4 were urban university-affiliated family medicine residency clinics and 3 were suburban, private pediatric clinics (staffed by approximately 130 and 11 physicians, respectively). Inclusion criteria included being a mother of a 0 - to 1-month-old infant who was registered at one of the participating clinics, being English literate, and being $\geq 12$ years old.

\section{Provider Training}

Providers from the participating practices were offered a 1-hour training session and printed educational materials about PPD and study procedures before the study's initiation. The training session covered the following topics: PPD background, risk factors, diagnosis, study protocol and methods, scoring of 2 depression screens (2-question screen and PHQ-9), and management, including components of stepped collaborative care, the role of the primary care provider, the use of antidepressants and psychotherapy, the selection of antidepressants for breastfeeding mothers, antidepressant side effects, the duration of treatment, the importance of social support, and indications for mental health referral.

\section{Measures}

Survey measures included (1) demographic information (initial survey), which indicated the mother's age, level of education, race/ethnicity, total family income, health insurance, marital status, number of children, and delivery date; (2) depression screening measures (all surveys), including a 2-question screen which asks about problems with mood or pleasure during the past month (positive if either question is answered "yes") and the PHQ-9 (positive if the score $>10)^{5}$; (3) receipt of treatment (9-month survey), which included the mother's self-report about being depressed or receiving a depression diagnosis after delivery, the mother's report of treatment in general and of antidepressant treatment and/or counseling in particular; (4) duration of treatment (9-month survey), including the 
number of weeks treated, treatment for $\geq 12$ weeks, and the number of counseling and psychiatry visits; (5) health outcomes (0- to 1-month and 9-month surveys), including the PHQ- $9,{ }^{5}$ the mental health scale (5 items, from SF-36) ${ }^{24}$ general health (single item from Short Form-36), the number of mother's and infant's days of illness during 2 weeks, and the number of mother's and infant's acute care visits to clinic/urgent care/emergency department during 2 weeks; (6) work outcomes (9-month survey), including the length of maternity leave, the number of hours missed at work during the previous 7 days, the number of hours worked during the previous 7 days, and the impact of health problems on activities unrelated to their job (work-related questions taken from the Work Productivity and Impairment Questionnaire $)^{25}$; and (7) satisfaction with depression treatment (9-month survey).

\section{Depression Diagnosis}

All participants were expected to complete the depression module of the SCID interview ${ }^{23}$ by telephone within 2 weeks of completing their 0- to 1-month survey, and again later if a previously nondepressed woman was screen-positive on a follow-up survey. The SCID interview, our reference standard for the diagnosis of major depressive disorder, was conducted by 3 trained psychology doctoral students whose training consisted of observing SCID training tapes and completing 5 practice tapes under the supervision of an experienced doctoral-level clinical psychologist, followed by weekly quality assurance assessment conferences throughout the study. Sixty-eight (13.4\%) of the women could not be reached for the initial SCID and an additional 9 women could not be reached for a follow-up SCID interview.

SCID-positive depressed participants were informed of the depression diagnosis, and these women were advised to see their primary care provider within 1 week. Providers were informed about their patients' depression diagnoses, the nature of the study, and their patients' participation in it. Although 31 of the randomized patients were each seen by a different provider, the remaining 8 patients shared providers: 3 providers had one patient in each of the 2 treatment groups, and one provider had 2 patients in the control group. Participants were also given the opportunity to selfdiagnose depression through a 9-month survey question with a yes/no answer: "Since your baby was born, have you been depressed or diagnosed with depression?"

\section{Randomization}

Women who became SCID-positive at 0 to 6 months postpartum were randomized to stepped or usual care using computer-generated block randomization schedules (block size, 10), which were stratified by clinic. This was done to ensure an approximately equal split of patients in each clinic. Providers were not blinded to their patients' group assignment.

\section{Intervention}

The stepped care intervention included (1) referral to the primary care provider for initial treatment (we recommended an antidepressant and/or psychotherapy referral, both found to be effective in previous research $\left.{ }^{21}\right)$; (2) regular telephone follow-up with a care manager (see details below); (3) decision support for primary care providers (eg, advice regarding specific antidepressants, additional treatment, or mental health referral); (4) consultation or referral to a mental health specialist for complex cases (eg, psychiatrists conducted psychiatric evaluations and adjusted medications and therapists provided psychotherapy using cognitive behavioral therapy, interpersonal therapy, or other therapies depending on the needs of the patient); and (5) patient education provided through the primary physician, care manager, and a mailed PPD brochure. Stepped care treatment was continued until either the patient was in remission, using Dietrich et al's ${ }^{8}$ definition of remission as a PHQ-9 score of $<5$, or the patient had passed the 9-month follow-up period. After this point, depression care continued according to the provider's standard.

The care manager was a registered nurse with mental health experience. She was trained in telephone call procedures by the principle investigator (DG), and training included PHQ-9 administration, depression diagnosis and treatment, patient education and social support, the use of the telephone protocol form, and triage of women with suicidal ideation.

Women in the stepped care group received an average of 4.1 calls from the care manager. These calls, which usually lasted 20 to 30 minutes, were attempted every 2 weeks until symptom remission and addressed depressive symptoms (using PHQ9), mental health visits, treatment adherence and 
side effects, social support, suicidal ideation/plans, and lifestyle issues including nutrition, exercise, and rest. The content of each call was documented on a form and a copy was faxed to the woman's primary provider. If a participant's symptoms were not resolving as expected, this was specifically communicated.

If a call or survey revealed suicidal ideation (for any participant), the principle investigator was informed and contacted the participant to determine the severity of her symptoms. For cases in which there was potential risk for injury or suicide (5 stepped care women, 1 control woman, and 1 SCID-negative woman), a plan of action was developed and executed that included informing the woman's provider and recommending an urgent visit to the primary care and/or mental health provider or emergency department. Also, if warranted and if the mother agreed, the principle investigator communicated with an adult friend or relative the need for 24-hour supervision of the mother until she was determined to be safe by a health professional. Providers were responsive to our communication about urgent problems and worked with the principle investigator in developing and implementing a plan of action. Control group women were also informed of their depression diagnosis and referred to their primary care provider, who managed the depression according to the provider's usual practice.

\section{Statistical Analysis}

To control for experiment-wide error rate on multiple dependent variables, 4 separate multivariate analyses of variance (MANOVAs) were performed to assess the impact of treatment on receipt of treatment, duration of treatment, health outcomes, and work outcomes (see "Measures," above, for a listing of variables included within each of these 4 categories). Independent variables were treatment group and specialty (family medicine versus pediatric clinic). If a given MANOVA was significant, individual ANOVAs were examined, looking at dependent variables within the group.

To determine the impact of the intervention on specific 9-month health, work, and duration of treatment outcomes, we performed one-way ANOVA (or $\chi^{2}$ tests) on randomized women with treatment group as the independent variable, and the following as dependent variables: PHQ-9 score, mental health score, general health score, mother's and infant's days of illness and number of acute care visits, length of maternity leave, hours spent at work during the previous week, hours of missed work during the previous week, the impact of health problems on work productivity and on regular activities (not related to work), the duration of treatment, treatment duration of $>12$ weeks, and the number of counseling and psychiatry visits.

A post hoc analysis was performed to compare 9-month health outcomes between women with self-diagnosed depression (both treated and untreated) versus women with no depression. To compare these 3 groups while controlling the experiment-wise error rate, we initially performed a multivariate analysis of covariance (MANCOVA) with the 3 "as-treated" groups and specialty as independent variables; the baseline (0 to 1 month) measure of the dependent variable as the covariate; and PHQ-9 score, mental health score, general health score, mother's and infant's days of illness, mother's and infant's health care visits, and activities not related to work as dependent variables. Given that the MANCOVA result was significant, we then performed univariate ANCOVAs on the above-listed dependent variables. Group comparisons on job-related outcomes could not be effectively evaluated because of the small number of depressed women who were at work at 9 months. A descriptive analysis was performed to identify reasons that self-diagnosed depressed women did not receive treatment.

$T$ tests and $\chi^{2}$ tests were used to compare stepped care and control patients on baseline demographic and health characteristics and to compare drop-outs (women who did not complete the final survey) with those who completed the study on selected variables.

\section{Results}

\section{Participants' Characteristics}

A total of 506 women participated in the study, which represented approximately $33 \%$ of the estimated 1556 eligible (English literate) women. Figure 1 illustrates the progress of women through the phases of the study.

Sixty-seven percent of participants were white, $18 \%$ were black, and $7 \%$ were Asian; $27 \%$ had annual family incomes of $<\$ 20,000$ whereas $37 \%$ had incomes of $\geq \$ 80,000$. Sixteen percent had less than a high school diploma and $52 \%$ had a 4 -year 


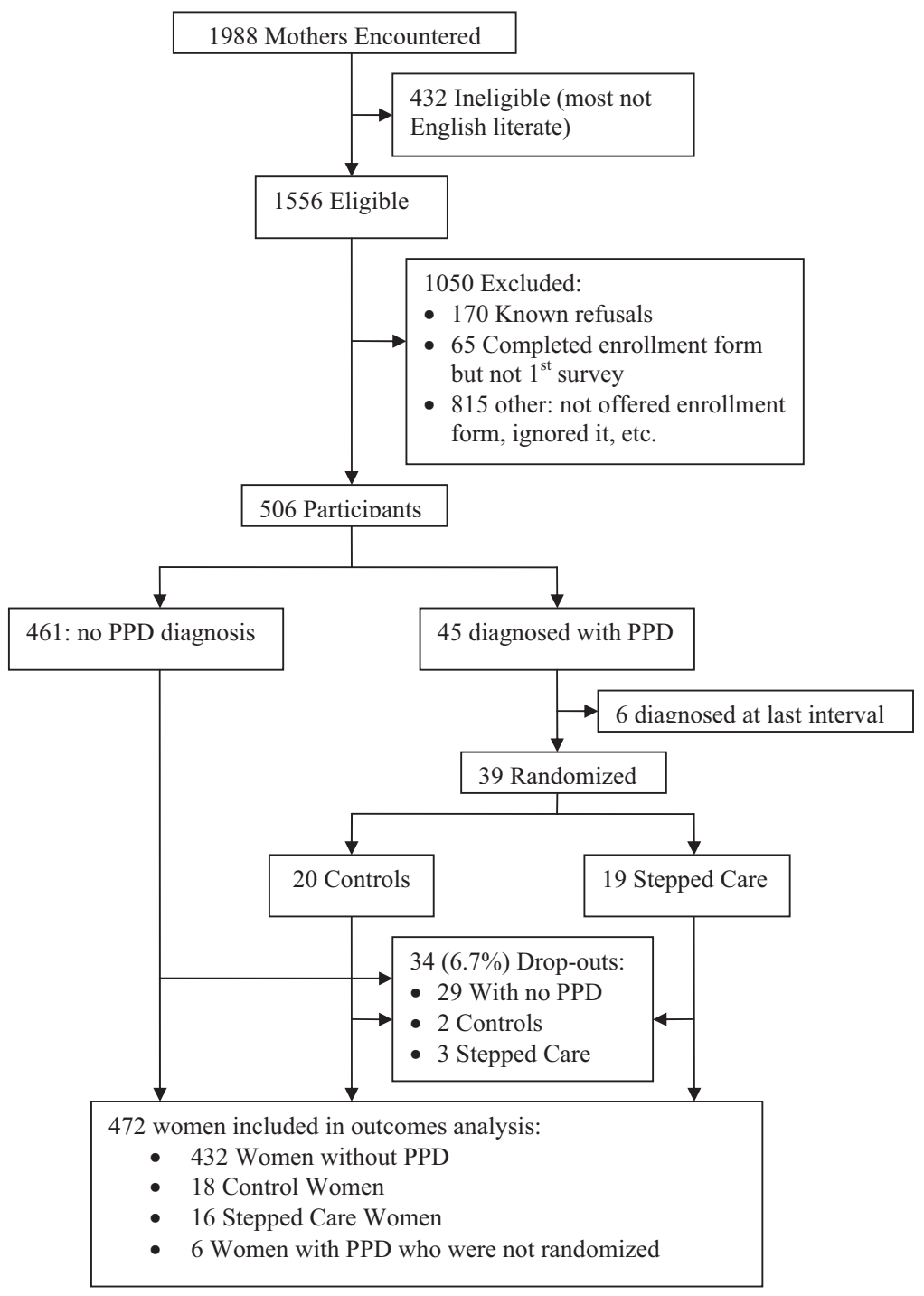

Figure 1. Flow diagram of participants' progress through phases of the study. PPD, postpartum depression.

degree or more education; $55 \%$ had private health insurance whereas $31 \%$ had medical assistance. The mean age of the mothers was 29.1 years (SD, 6.2 ; range, $16-46) ; 65 \%$ of mothers were married, $64 \%$ were employed, and $42 \%$ had only one child. One hundred sixty-seven (33\%) participants were recruited from family medicine clinics and 339 (67\%) from pediatric clinics. More detail about demographic characteristics was provided in a previous publication. ${ }^{26}$

Only $34(6.7 \%)$ of the 506 participants did not complete the final survey and were therefore considered dropouts. When dropouts were compared with those who completed the study, dropouts were found to be younger (26 vs 29 years old), less educated (32\% vs $73 \%$ had more than a high school education), were less likely to be married (27\% vs $68 \%$ ), had more children (2.5 vs 1.9$)$, had lower family incomes $(64 \%$ vs $25 \%$ had annual income $<\$ 20,000)$, and had more depressive symptoms (21\% vs $6 \%$ had a positive PHQ-9 score). ${ }^{26}$

\section{SCID-Positive Depression}

Forty-five women (8.9\%) had SCID-positive depression: 19 women were randomized to stepped care (the intervention group), 20 women were randomized to usual care (the control group), and 6 women were diagnosed at the 9-month interval and therefore could not be randomized. Thirty-four of the 39 randomized women completed the final survey. Baseline demographic and health characteristics of this group indicate that a 
Table 1. Baseline Comparisons between Stepped Care and Control Subjects on Demographic and Health Characteristics

\begin{tabular}{|c|c|c|c|}
\hline Characteristics & Stepped Care $(\mathrm{n}=19)$ & Control $(\mathrm{n}=20)$ & $P^{*}$ \\
\hline Age (mean yrs $[\mathrm{SD}]$ ) & $27.2(5.2)$ & $28.0(7.3)$ & .717 \\
\hline$\leq$ High school education (n [\%]) & $10(52.6)$ & $12(60.0)$ & .843 \\
\hline Non-white (n [\%]) & $11(57.9)$ & $13(65.0)$ & .748 \\
\hline Total family income $<\$ 40,000$ (n [\%]) & $16(84.2)$ & $13(65.0)$ & .278 \\
\hline Receiving medical assistance (n [\%]) & $15(83.3)$ & $9(52.9)$ & .075 \\
\hline Married (n [\%]) & $5(26.3)$ & $8(40.0)$ & .501 \\
\hline Number of children (mean [SD]) & $2.8(1.4)$ & $2.2(1.1)$ & .204 \\
\hline $\begin{array}{l}\text { Patient Health Questionnaire-9 score } \\
(\text { mean }[\mathrm{SD}])^{\dagger}\end{array}$ & $10.5(8.5)$ & $11.7(7.2)$ & .652 \\
\hline Mental health score (mean $[\mathrm{SD}])^{\ddagger}$ & $18.1(6.3)$ & $18.0(5.8)$ & .978 \\
\hline General health score (mean $[\mathrm{SD}])^{\S}$ & $2.9(0.9)$ & $3.2(0.8)$ & .253 \\
\hline \multicolumn{4}{|l|}{ Number of illness days (mean [SD]) } \\
\hline Mother & $1.9(4.2)$ & $2.5(4.8)$ & .728 \\
\hline Infant & $0.7(3.2)$ & $0.4(1.6)$ & .639 \\
\hline \multicolumn{4}{|l|}{ Number of acute care visits (mean [SD]) } \\
\hline Mother & $0.3(0.7)$ & $0.6(2.0)$ & .631 \\
\hline Infant & $0.1(0.5)$ & $0(0)$ & .331 \\
\hline
\end{tabular}

*Determined by $\chi^{2}$ and $t$ tests. Percentages are expressed as the percent of the given treatment group.

${ }^{\dagger}$ Patient Health Questionnaire-9 score range $=0-27$, with higher numbers representing more depressive symptoms.

${ }^{\ddagger}$ Mental Health score range $=5-30$, with higher numbers representing better mental health.

${ }^{\S}$ General health, single item: $1=$ poor, $2=$ fair, $3=$ good, $4=$ very good, $5=$ excellent.

majority had low education and income levels and most were not white and were receiving medical assistance. No significant baseline differences were seen between the 2 treatment groups, indicating that the randomization procedure was effective (Table 1).

Women in both the stepped care and control groups were generally satisfied with their treatment, with a mean (SD) satisfaction score of 4.4 (1.6) for the stepped care group, and 5.5 (1.2) for the control group (satisfaction scale: $1=$ very dissatisfied, $4=$ somewhat satisfied, $7=$ very satisfied; $P=.091)$. Women in the stepped care group were receptive to follow-up calls by the care manager and they each received an average of 4.1 calls (range, 0-11). Only one stepped care participant could not be reached for follow-up calls.

\section{Impact of Intervention on Outcomes}

MANOVA results (with treatment group and specialty as independent variables) showed that the stepped care intervention had a significant positive impact on the receipt of treatment $(\lambda=$ $0.738 ; \mathrm{F}=3.305 ; P=.035)$. Results from oneway ANOVAs on these individual outcomes are shown on Table 2 and indicate that the intervention improved mothers' awareness of their depression diagnosis and their receipt of treatment, particularly antidepressant medication. For the remaining 3 MANOVAs (duration of treatment,

Table 2. The Impact of Stepped Care on Structured Clinical Interview for DSM-IV-Positive Depressed Patients' Self-Report of Depression and Receipt of Treatment

\begin{tabular}{lcrr}
\hline Outcome (n [\%]) & Stepped Care (n = 16) & Control (n $=18)$ & $P^{*}$ \\
\hline Self-reported depression after delivery & $16(100)$ & $11(61.1)$ & .008 \\
Received treatment (antidepressants, psychotherapy) & $15(93.8)$ & $10(55.6)$ & .019 \\
Received antidepressants & $15(93.8)$ & $10(55.6)$ & .019 \\
Received counseling & $7(43.8)$ & $5(27.8)$ & 1.00 \\
\hline
\end{tabular}

*Determined by one-way analysis of variance. 
Table 3. Impact of Stepped Care Treatment for Postpartum Disorder on 9-Month Health, Work, and Duration of Treatment Outcomes

\begin{tabular}{|c|c|c|c|}
\hline Outcomes & Stepped Care $(\mathrm{n}=16)$ & Control $(\mathrm{n}=18)$ & $P^{*}$ \\
\hline PHQ-9 score $^{\dagger}$ & $9.0(7.3)$ & $7.6(6.5)$ & .597 \\
\hline Women with positive PHQ-9 (score $\geq 10$ ) (n [\%]) & $7(44)$ & $5(28)$ & .475 \\
\hline Mental Health score ${ }^{\ddagger}$ & $18.8(5.9)$ & $20.7(5.4)$ & .356 \\
\hline General Health score ${ }^{\S}$ & $2.8(1.0)$ & $2.8(0.6)$ & .851 \\
\hline \multicolumn{4}{|l|}{ Number of illness days (within 2 weeks) } \\
\hline Mother & $3.3(4.9)$ & $2.6(5.0)$ & .869 \\
\hline Infant & $1.1(3.5)$ & $1.9(3.0)$ & .466 \\
\hline \multicolumn{4}{|l|}{ Number of clinic/urgent care visits (within 2 weeks) } \\
\hline Mother & $0.2(0.8)$ & $0.2(0.04)$ & .972 \\
\hline Infant & $0.1(0.3)$ & $0.6(1.9)$ & .407 \\
\hline Length of maternity leave (weeks) ${ }^{\|}$ & $23.0(12.7)$ & $9.1(4.8)$ & .024 \\
\hline Number of hours spent at work during past week & $34.0(2.8)$ & $35.0(7.2)$ & .861 \\
\hline Number of hours of missed work during past week & $4.0(5.7)$ & $1.5(2.1)$ & .296 \\
\hline Impact of health problems on work productivity ${ }^{\text {II }}$ & $1.0(1.4)$ & $2.0(2.4)$ & .604 \\
\hline Impact of problems on regular activities ${ }^{\mathbb{I}}$ & $3.9(3.1)$ & $2.4(2.8)$ & .562 \\
\hline Number of weeks of treatment & $19.8(11.5)$ & $19.6(12.8)$ & .363 \\
\hline Women treated for $\geq 12$ weeks (n $[\%]$ ) & $9(75)$ & $4(67)$ & .252 \\
\hline Number of counseling visits & $5.3(8.8)$ & $2.3(3.1)$ & .131 \\
\hline Number of psychiatry visits & $0.8(2.1)$ & $0.5(0.8)$ & .928 \\
\hline
\end{tabular}

All values presented as mean (SD) unless otherwise indicated.

*Determined by one-way analysis of variance or $\chi^{2}$.

${ }^{\dagger}$ PHQ-9 score range $=0$ to 27 , with higher numbers representing more depressive symptoms.

${ }^{\ddagger}$ Mental Health score range $=5$ to 30 , with higher numbers representing better mental health.

${ }^{\$}$ General health, single item: 1 = poor, $2=$ fair, $3=$ good, $4=$ very good, $5=$ excellent.

"The analysis on length of leave included only 2 stepped care subjects and 8 control subjects.

IImpact of health problems on work productivity or regular (non-job related) activities: scale of 0 to 10, where higher numbers represent greater negative health impact.

PHQ, Patient Health Questionnaire.

health outcomes, and work outcomes), treatment group effects were not significant. Group comparisons on specific health, work, and treatment duration outcomes are given on Table 3; with one exception (length of maternity leave), there were no significant group differences.

Specialty effects approached statistical significance in 2 MANOVAs: receipt of treatment $(\lambda=$ $0.771 ; \mathrm{F}=2.769 ; P=.060)$ and health outcomes $(\lambda=0.594 ; \mathrm{F}=2.346 ; P=.056)$. In both cases, mothers whose infants attended pediatric clinics had better outcomes than those who attended family medicine clinics. There were no significant group by specialty interactions.

\section{Self-Diagnosed Depression}

When patients were asked if they had been depressed or diagnosed with depression since delivery, $122(24.1 \%)$ answered "yes," with 76 (62\%) reporting treatment and $46(38 \%)$ no treatment.
Three-fourths of women (344) said they had not been depressed.

MANCOVA results showed that these 3 selfdiagnosis groups were significantly different in their health outcomes $(\lambda=.726 ; \mathrm{F}=11.139 ; P=$ .000). Follow-up ANCOVAs found that depressed women (both treated and untreated) compared with nondepressed women had more depressive symptoms (PHQ-9), worse general health and mental health, and greater impact of health problems on regular activities. Treated depressed women, versus the other 2 groups, had more acute care visits (Table 4). Specialty effects and specialty/ group interactions were also significant $(\lambda=0.929$ and $0.891 ; \mathrm{F}=4.9$ and $3.8 ; P=.000$ and .000 , respectively); mothers from family medicine clinics, particularly those with treated self-diagnosed depression, had worse outcomes. Women's reasons for not taking depression treatment are shown in Table 5. 
Table 4. Significant Differences in 9-Month Health Outcomes between the Treated Depressed Women, Untreated Depressed Women, and Nondepressed Women Self-Report Groups

\begin{tabular}{|c|c|c|c|c|c|}
\hline Health Outcomes & $\begin{array}{l}\text { Depressed/Treated } \\
\quad(\mathrm{n}=76)\end{array}$ & $\begin{array}{l}\text { Depressed/Untreated } \\
\quad(\mathrm{n}=46)\end{array}$ & $\begin{array}{l}\text { Nondepressed } \\
\quad(\mathrm{n}=344)\end{array}$ & $\mathrm{F}$ & $P^{\star}$ \\
\hline PHQ-9 score ${ }^{\dagger}$ & $7.9(6.2)$ & $7.3(5.1)$ & $2.3(2.8)$ & 53.7 & .000 \\
\hline Mental health score ${ }^{\ddagger}$ & $20.6(4.9)$ & $21.3(4.2)$ & $25.6(2.9)$ & 68.8 & .000 \\
\hline General health score ${ }^{\S}$ & $3.0(0.9)$ & $3.3(0.7)$ & $3.8(0.9)$ & 129.6 & .000 \\
\hline \multicolumn{6}{|l|}{ Number of illness days (within 2 weeks) } \\
\hline Mother & $3.3(6.0)$ & $2.3(4.4)$ & $1.3(3.1)$ & 3.7 & .054 \\
\hline Infant & $1.5(3.1)$ & $1.6(3.2)$ & $1.1(2.6)$ & 0.1 & .820 \\
\hline \multicolumn{6}{|l|}{ Number of acute care visits (within 2 weeks) } \\
\hline Mother & $0.3(1.5)$ & $0.1(0.4)$ & $0.1(0.5)$ & 35.1 & .000 \\
\hline Infant & $0.4(1.8)$ & $0.2(0.5)$ & $0.2(0.5)$ & 0.5 & .499 \\
\hline Impact of problems on regular activities" & $3.2(2.9)$ & $2.0(2.5)$ & $1.0(1.8)$ & 14.7 & .000 \\
\hline
\end{tabular}

All values provided as mean $(\mathrm{SD})$.

*Determined by analysis of covariance.

${ }^{\dagger}$ PHQ-9 score range $=0-27$, with higher numbers representing more depressive symptoms.

${ }^{\ddagger}$ Mental health score range $=5-30$, with higher numbers representing better mental health.

${ }^{\S}$ General health, single item: 1 = poor, $2=$ fair, 3 = good, $4=$ very good, $5=$ excellent.

"Impact of health problems on regular activities (unrelated to job), scale of $0-10$, with higher numbers representing greater negative health impact.

PHQ, Patient Health Questionnaire.

\section{Discussion}

Our stepped care intervention improved mothers' awareness of their depression diagnosis and their likelihood of being treated-both important clinical outcomes. The stepped care intervention did

Table 5. Mothers' Reasons for Not Taking Depression Treatment*

\begin{tabular}{lc}
\hline Reason & $\begin{array}{c}\text { Mothers } \\
(\mathrm{n}[\%])^{\dagger}\end{array}$ \\
\hline $\begin{array}{l}\text { Mother thought she could handle it herself } \\
\text { No time for depression visits }\end{array}$ & $21(46)$ \\
$\begin{array}{l}\text { Treatment not recommended } \\
\text { Concerned about what others would think; } \\
\text { embarrassed }\end{array}$ & $11(35)$ \\
$\begin{array}{l}\text { Concerned about medication effects on nursing } \\
\text { infant }\end{array}$ & $8(17)$ \\
$\begin{array}{l}\text { Concerned about medication side effects on self } \\
\text { Thought it was "just a phase" or a short-term } \\
\text { problem }\end{array}$ & $6(13)$ \\
$\begin{array}{l}\text { No childcare available during depression visits } \\
\text { Concerns about insurance and cost of coverage }\end{array}$ & $5(11)$ \\
Concerned about becoming dependent on & $4(9)$ \\
$\quad$ medications & $3(7)$ \\
$\begin{array}{l}\text { Husband/partner unsupportive of her getting help } \\
\text { Worried that baby would be taken away }\end{array}$ & $1(2)$ \\
\hline
\end{tabular}

*Self-diagnosed women $(\mathrm{n}=46)$.

${ }^{\dagger}$ Results show women who reported that they had been depressed but not treated. not, however, seem to significantly impact treatment duration, health, or work outcomes. One explanation for the absence of observed impact on these outcomes is the relatively small number of women available for these analyses: 18 for duration of treatment, 34 for health outcomes, and 10 for work outcomes. Other factors that may have contributed to nonsignificant group differences are the relatively brief period of follow-up and the inclusion of women with chronic depression and other associated severe mental health conditions (eg, bipolar disease), who may have been less responsive to this treatment program. In addition, the larger number of presumably higher-risk medical assistance patients in the stepped care group (15 vs 9; $P=.075)$ may have blunted intervention effects.

It is interesting that women's self-reported diagnosis of depression produced nearly 3 times the number of depressed cases as the SCID-based diagnoses (122 vs 45, respectively). This large discrepancy between self-reported and SCIDbased diagnoses is probably caused by the nature of our measures, at least in part. For example, the SCID is designed to diagnose major depression whereas the self-report question may have also identified women with transient or subthreshold depressive symptoms. We may have also missed cases because of noncompletion of a survey or 
SCID interview or discordance in survey timing with depressive symptoms. We are not aware of other studies comparing mothers' self-reported depression to criterion-based depression, and the term "self-reported depression" in other PPD studies usually refers to positive depression surveys. ${ }^{27-30}$ This unexpected finding should be pursued in future studies.

As anticipated, our analysis comparing women from the 3 self-report groups (depressed/treated, depressed/untreated, and nondepressed) revealed that depressed mothers have worse mental and physical health outcomes and that depressed women's health problems have a significantly greater impact on activities not related to their jobs. Because caring for one's infant is usually a large component of a mother's "regular" activities, it follows that depression probably impacts a mother's care of her newborn. Prior research has also shown a relationship between mothers' and fathers' depressive symptoms. ${ }^{31-33}$ Therefore, it is important that depressed women be effectively treated, both for their own sake and the sake of other family members.

Importantly, 46 (38\%) of the self-diagnosed depressed women said they had not received treatment. Although the most common reason for not receiving treatment was that women thought they could handle their depression themselves, postpartum-specific concerns were also raised, including medication effects on nursing infants, lack of childcare during depression visits, no time for visits, or fear that the infant might be taken away. Interventions that address these treatment barriers (eg, nurse phone calls exploring reasons for not receiving treatment, patient and physician education about treatment risks and benefits, or cultural liaisons for women of diverse cultural/ethnic backgrounds) should be explored in future studies.

Observed specialty effects-worse outcomes for women from family medicine (vs pediatric) siteswere confounded by other factors. The family medicine clinics were all urban residency training sites whereas the pediatric clinics were suburban community-based clinics. Therefore, our specialtybased differences could be based on socioeconomic factors.

Strengths of the study included case finding during well-child visits in pediatric and family medicine clinics; a low drop-out rate; depression diagnoses based on a reference standard (SCID interview); quantitative comparison of SCID-based depression diagnosis to self-diagnosis; the use of randomized controlled trial design with a practical, stepped, collaborative care intervention; and our finding that the intervention positively impacted depressed mothers' knowledge of their diagnosis and their treatment. Weaknesses included our lower-than-expected participation rate, specialty effects being intertwined with residency training and socioeconomic characteristics, the modest number of SCID-diagnosed women, and, as a result, the apparent absence of treatment effect on health outcomes. Future studies exploring this intervention with larger numbers of depressed women would be useful.

\section{Conclusion}

This study found that the stepped care intervention increased mothers' recognition of their depression diagnosis and improved rates of treatment. In addition, formal diagnostic procedures (eg, SCID interviews) missed many women whose depressed mood interfered with their psychological health and daily functioning.

\section{References}

1. Gaynes BN, Gavin N, Meltzer-Brody S, et al. Perinatal depression: prevalence, screening accuracy, and screening outcomes. Evidence Report/Technology Assessment no. 119. AHRQ Publication .o. 05E006-2. Rockville, MD: Agency for Healthcare Research and Quality; 2005.

2. Kelly BD. St John's wort for depression: what's the evidence? Hosp Med 2001;62:274-6.

3. Seehusen DA, Baldwin LM, Runkle GP, Clark G. Are family physicians appropriately screening for postpartum depression? J Am Board Fam Med 2005; 18:104-12.

4. Spitzer RL, Kroenke K, Williams JBW, et al. Validation and utility of a self-report version of PRIMEMD: the PHQ primary care study. JAMA 1999;282: 1737-44.

5. Kroenke K, Spitzer RS, Williams JBW. Validity of a brief depression severity measure. J Gen Intern Med 2001;16:606-13.

6. Hedrick SC, Chaney EF, Felker B, et al. Effectiveness of collaborative care depression treatment in Veterans' Affairs primary care. J Gen Intern Med 2003;18:9-16.

7. Hunkeler EM, Katon W, Tang L, et al. Long term outcomes from the IMPACT randomized trial for depressed elderly patients in primary care. BMJ 2006;332:259-63.

8. Dietrich AJ, Oxman TE, Burns MR, Winchell CW, Chin T. Application of a depression management 
office system in community practice: a demonstration. J Am Board Fam Pract 2003;16:107-14.

9. Katon W, Von Korff M, Lin E, et al. Collaborative management to achieve treatment guidelines. Impact on depression in primary care. JAMA 1995;273: 1026-31.

10. Unutzer J, Katon W, Callahan CM, et al. Collaborative care management of late-life depression in the primary care setting. JAMA 2002;288:2836-45.

11. Oxman TE, Dietrich AJ, Schulberg HC. The depression care manager and mental health specialist as collaborators within primary care. Am J Geriatr 2003;11:507-16.

12. Gilbody S, Bower P, Fletcher J, Richards D, Sutton AJ. Collaborative care for depression: a cumulative meta-analysis and review of longer-term outcomes. Arch Intern Med 2006;166:2314-21.

13. Katon W, Unutzer J, Fan MY, et al. Cost-effectiveness and net benefit of enhanced treatment of depression for older adults with diabetes and depression. Diabetes Care 2006;29:265-70.

14. Lin EH, Katon W, Von Korff M, et al. Effect of improving depression care on pain and functional outcomes among older adults with arthritis: a randomized controlled trial. JAMA 2003;290:2428-9.

15. Callahan CM, Kroenke K, Counsell SR, et al. Treatment of depression improves physical functioning in older adults. J Am Geriatr Soc 2005;53:367-73.

16. Rost K, Smith JL, Dickinson M. The effect of improving primary care depression management on employee absenteeism and productivity: a randomized trial. Med Care 2004;42:1202-10.

17. Schoenbaum M, Unutzer J, Sherbourne C, et al. Cost-effectiveness of practice-initiated quality improvement for depression. JAMA 2001;286:1325-30.

18. Whisman MA. Marital adjustment and outcome following treatments for depression. J Consult Clin Psychol 2001;69:125-9.

19. Katon W, Von Korff M, Lin E, Simon G. Rethinking practitioner roles in chronic illness: the specialist, primary care physician, and the practice nurse. Gen Hosp Psychistry 2001;23:138-44.

20. Ugarriza DN. Group therapy and its barriers for women suffering from postpartum depression. Arch Psychiatr Nurs 2004;18:39-48.

21. Gjerdingen D. The effectiveness of various postpartum depression treatments and the impact of antidepressant drugs on nursing infants. J Am Board Fam Pract 2003;16:372-82.

22. Heneghan AM, Mercer MB, Deleone NL. Will mothers discuss parenting stress and depressive symptoms with their child's pediatrician? Pediatrics 2004;113:460-7.

23. First MG, Spitzer RL, Gibbon M, Williams JB. Structured Clinical Interview for DSM-IV Axis I Disorders. Clinical version, administration booklet. New York: Biometrics Research Department, New York State Psychiatric Institute; 1997.

24. Ware JE, Snow KK, Kosinski M, Gandek B. SF-36 Health Survey: manual and interpretation guide. Boston: The Health Institute, New England Medical Center; 1993.

25. Reilly MC, Zbrozek AS, Dukes EM. The validity and reproducibility of a work productivity and activity impairment instrument. Pharmacoeconomics 1993;4:353-65.

26. Gjerdingen D, Crow S, McGovern P, Miner M, Center B. Postpartum depression screening at wellchild visits: validity of a 2-question screen and PHQ-9. Ann Fam Med 2009;7:63-70.

27. Wenzel A, Gorman LL, O'Hara MW, Stuart S. The occurrence of panic and obsessive compulsive symptoms in women with postpartum dysphoria: a prospective study. Arch Women Ment Health 2001;4: $5-12$.

28. Centers for Disease Control and Prevention. Prevalence of self-reported postpartum depressive symptoms-17 states, 2004-2005. MMWR Morb Mortal Wkly Rep 2008;57:361-6.

29. LaRocco-Cockburn A, Melville J, Bell M, Katon W. Depression screening attitudes and practices among obstetrician-gynecologists. Obstet Gynecol 2003; 101:892-8.

30. Muzik M, Klier CM, Rosenblum KL, Holzinger A, Umek W, Katschnig H. Are commonly used selfreport inventories suitable for screening postpartum depression and anxiety disorders? Acta Psychiatr Scand 2000;102:71-3.

31. Morse CA, Buist A, Durkin S. First-time parenthood: influences on pre- and postnatal adjustment in fathers and mothers. J Psychosom Obstet Gynaecol 2000;21:109-20.

32. Pinheiro RT, Magalhaes PV, Horta BL, Pinheiro KA, da Silva RA, Pinto RH. Is paternal postpartum depression associated with maternal postpartum depression? Population-based study in Brazil. Acta Psychiatr Scand 2006;113:230-2.

33. Roberts SL, Bushnell JA, Collings SC, Purdie GL. Psychological health of men with partners who have post-partum depression. Aust N Z J Psychiatry 2006; 40:704-11. 